\title{
Kurzbeitrag
}

\author{
Manfred Körber, Jolanda Staman
}

\section{Der Wandel der Arbeitsgesellschaft im Spiegel aktueller sozialethischer Texte}

"Wir müssen lernen, die nicht verwirklichten Möglichkeiten zu erkennen, die Chancen, die sich in den Rissen unserer zerfallenden Gesellschaft verbergen." Mit diesem Appell beginnt André Gorz sein Buch über die in der Krise der Arbeitsgesellschaft liegenden Herausforderungen.

Angesichts des Anstiegs der Arbeitslosenzahlen in den letzten zwei Jahrzehnten gibt es nicht nur in den Sozialwissenschaften eine intensive Diskussion um die Zukunft der Arbeitsgesellschaft. Wird die Vollbeschäftigungsgesellschaft wieder möglich; ist sie aus ökologischen Gründen wünschenswert, oder wird die Zukunft der Arbeit jenseits der Erwerbsarbeit liegen? So lauten die Fragestellungen, auf die es höchst unterschiedliche Antworten gibt. Auffallend ist, dass es zu dieser Diskussion nur wenige sozialethische Beiträge gibt.

Anders als in den 80er Jahren, als die Massenarbeitslosigkeit zu einer breiten sozialethischen Diskussion um die Zukunft der Arbeit führte, wird dem Thema heute kaum Beachtung geschenkt. Sozialethische Positionen werden vielmehr implizit oder ausdrücklich in den unterschiedlichen Analysen und Handlungskonzepten der Sozialwissenschaft vorgetragen. Gerade die Veröffentlichung des Sozialwortes der beiden großen deutschen Kirchen, das eine klare Positionsbestimmung der Kirchen zur sich wandelnden Arbeitsgesellschaft vorbringt (vgl. Sozialwort 1997 zum Stichwort "Arbeit") oder die brisanten Ergebnisse der sächsisch-bayerischen Zukunftskommission, erfordern u.E. allerdings eine sozialethische Debatte.

Die folgenden Überlegungen wollen einen Anstoß dazu liefern, indem sie eine Interpretation für die mangelnde Beachtung der Diskussion um die Krise der Arbeitsgesellschaft durch die Sozialethik anbieten. Dazu stellen wir die wichtigsten Beiträge der letzten 10 Jahre, ihre Problemwahrnehmung und sozialethische Positionierung vor. Wir gehen dabei thematisch vor, indem wir zunächst auf den Wandel menschlichen Arbeitens eingehen (1.), bevor wir
Maßstäbe für eine sozialethische Einmischung in den Wandel der Arbeitsgesellschaft entwickeln und vorliegende Handlungsansätze anhand dieser Maßstäbe überprüfen (2.).

\section{$1 \quad$ Menschliches Arbeiten im Wandel}

\subsection{Werteverschiebungen}

Arbeit erschöpft sich nicht in Erwerbsarbeit, darin sind sich die Mehrheit der sozialethisch arbeitenden Autorinnen und Autoren einig. Zur Herleitung des Arbeitsbegriffes wird häufig schöpfungstheologisch argumentiert: Der Mensch gilt im zweiten Schöpfungsbericht nach dem Sündenfall als zur Strafe Arbeitender "im Schweiße seines Angesichtes sollst du..."(Gen 3, 17; Zimmerli 1997, 147; Guggenberg 1999, 125). Möhring-Hesse resümiert, dass dieser theologische Begriff der Arbeit nicht auf die gegenwärtig dominante Form der Arbeit, nämlich die Erwerbsarbeit eingestellt ist. Das neuzeitliche Arbeitsverständnis löste sich von der "theologisch vormals aufgegebene(n) "Mühe, Qual und Last" (und) wurde zur kreativen und autonomen Tätigkeit, durch die Menschen eigene Bedürfnisse befriedigen, eine selbständige Existenz erlangen und sich zu autonomen Persönlichkeiten entwickeln.“(MöhringHesse 2000, 82) Heute wird von verschiedenen Seiten ein entsprechend umfassendes humanes $\mathrm{Ar}$ beitsverständnis eingefordert (Hengsbach 1999, 14; Müller 1997, 82; EKD 1991, 22)

Sozialethische Ansätze zu einer Formulierung einer solchen "Leitvorstellung" finden sich z.B. bei Sander, der den inneren Zusammenhang von Arbeit und Leben bestimmt: Arbeit und Leben sind als Schöpfungstatsachen innerlich miteinander verbunden. Leben ist dabei eine Realität der Schöpfung und Arbeit die Technik, es zu erhalten. Arbeit ist eine Aktion des Lebens im Widerstand gegen den Tod (Sander 1996, 154ff.). Müller weist darauf hin, dass bereits die Enzyklika Centesimus Annus Arbeit als kulturelle Tätigkeit hervorgehoben hat. Mehr geistig-kulturelle Selbstentfaltung in der Arbeit ist eine Voraussetzung für die Entwicklung neuer Sozialtugenden wie Kreativität, Partizipation und Eigeninitiative (Müller 1997, 82). 
Die Konsequenz einer veränderten Sicht auf den Wert der Arbeit im Leben fasst Guggenberg (1999, 126f.) folgendermaßen zusammen: "Der Unterschied ist ungeheuer, ob die Welt vom Standpunkt der Kontemplation oder vom Standpunkt der Arbeit her erschlossen wird, ob der aktiv-arbeitsförmige Umgang mit der Welt oder das zuschauende Teilhaben an ihr den archimedischen Punkt der Welt- und Selbstinterpretation bezeichnet; ob die spezifische Kulturleistung des Menschen die Erzeugung der Sinnhaftigkeit des Daseins, den Rang der wichtigsten "Produktion" einnimmt, oder ob dieser Rang der Beschaffung von Brot und Wein, Haus und Herd, Waffe und Werkzeug zukommt." Guggenberg möchte vermieden sehen, dass "die Sache der Zukunft (...) konsequent als Zukunft der Sache beschreibbar (wird), als ein Problem der schieren Quantität, der durch Arbeit ständig zu erweiternden Güterfülle."

\subsection{Der Sinn menschlichen Tätigseins}

Können Sozialethiker von der vita contemplativa und dem Bau des "neuen Athen", von der Utopie eines arbeitsfreien Lebens reden, so heißt dies noch lange nicht, dass dies in einer vom Leistungsgedanken geprägten, nach arbeitsgesellschaftlichen Normen funktionierenden Gesellschaft auf Anerkennung stößt. Trotz des nicht wegzudiskutierenden Wertewandels im Bezug auf das Verständnis von Arbeit, ist der alte Menschheitstraum, "die Arbeit los“ zu sein, für große Teile der Gesellschaft (noch) nicht als Option für die jeweils eigene Zukunft denkbar. Noch wird das "Vollbringen" des Lebens auf das "Hervorbringen" in der Arbeit reduziert (Guggenberg 1999, 126). Wie sehr unsere Gesellschaft noch in den arbeitsgesellschaftlichen Strukturen verhaftet ist, lässt sich an der mangelnden gesellschaftlichen Integration, den Problemen für die Identitätsbildung, soziale, politische und sozialpsychologische Probleme von Erwerbsarbeitslosen ersehen (Ruh 1996, 205f.; Foitzik 1996, 55). Arbeitslosigkeit offenbart also die "schöpferische Macht der Arbeit für das Leben. Sie zeigt die innerliche Verbindung von Leben und Arbeit." (Sander 1996, 154). Jedoch können die Veränderungen als Herausforderung und Chance angenommen werden, grundsätzlich über das Verhältnis von Mensch und Arbeit nachzudenken.

Die Sinnhaftigkeit menschlicher Arbeit erfasst Honecker, indem er zwei Zielsetzungen annimmt: "Das kollektive Ziel der Arbeit ist die Erzeugung von Gütern und Dienstleistungen. Das individuelle Ziel ist, dass Arbeit dem Erwerb, dem Lebensunter- halt des Einzelnen und dessen Selbstverwirklichung dienen soll.“ Aber Würde, Lebenswert besitzt der Mensch vor aller Arbeit (Honecker 1995, 457, 461).

Für Hengsbach hat der subjektive Sinn menschlicher Arbeit drei Funktionen: Die menschliche Arbeit dient 1. der Selbsterhaltung (Naturalfunktion), sie dient 2. der Integration in die Gesellschaft und dient somit der Gesellschaft (Sozialfunktion). 3. Der personale Charakter der Arbeit dient als Maßstab zur Bewertung der Arbeit, nicht ihr objektiver Wert (Personalfunktion). (Hengsbach 1990, 27f.)

Mieth $(1999,87)$ steht mit Hengsbach in einer Argumentationslinie, wenn er - das Subjekt im Blick - von vier menschlichen Grundbedürfnissen ausgeht. Das Bedürfnis aus eigener Kraft Mittel zum Überleben und Besserleben bereitstellen zu können, deckt sich mit der Naturalfunktion Hengsbachs. Das von Mieth formulierte Bedürfnis nach sozialer Anerkennung deckt sich inhaltlich mit der Sozialfunktion Hengsbachs. Durch die Betonung des menschlichen Bedürfnisses nach persönlichen Beziehungen und das Bedürfnis nach Sinn menschlichen Tätigseins geht Mieth genauer auf die Personalfunktion menschlicher Tätigkeit ein. D.h., der Sinn menschlichen Tätigseins liegt primär in seiner personalen Funktion. Dabei unterscheidet Mieth zwischen drei menschlichen Tätigkeitsformen: Arbeit als Existenzsicherung, Spiel und Wirken. Das Wirken zeichnet sich durch Zeit-, Sach- und personale Souveränität aus. Im schöpferischen Wirken bildet der Mensch Gott ab, nicht in der Arbeit, denn Arbeit heißt Mühsal (Mieth 1999, 88).

Die Betonung der kollektiven Sinnstiftung menschlichen Tätigseins begegnet bei Sander und Ulrich. Sander erklärt: "Solidarität für die Güte des Lebens ist das entscheidende Merkmal des Schöpfungsgutes Arbeit“ (Sander 1996, 162). Ulrich verwendet den Richschen Begriff der "Lebensdienlichkeit“, wenn er vom Ziel des guten Lebens und des gerechten Zusammenlebens spricht (Ulrich 1997, 146).

\section{Maßstäbe für eine sozialethische Einmischung in den Wandel der Arbeitsgesellschaft}

\subsection{Politisch-ethische Maßstäbe}

Ausgehend von der Voraussetzung, dass der Mensch vor aller Arbeit Würde besitzt, werden in den vorliegenden sozialethischen Positionen Maßstäbe und Kriterien für die humane Gestaltung des Wandels der Arbeitsgesellschaft entwickelt. 
Der Mensch soll im Mittelpunkt der Wirtschaft stehen (Müller 1997, 81). Es gilt also das Personalprinzip, was neben dem Vorrang des tätigen Menschen auch den Vorrang des menschlichen Profils menschlicher Tätigkeit vor der Entwicklung der Dinge impliziert. "Die Definitionsmacht des Kapitalverwertungsprozesses für Investitionen und technologische Vorgänge muss zugunsten der Menschenwürde in der Tätigkeit eingeschränkt werden." (Mieth 1999, 95) Zur Wahrung des Personalprinzips entwickelt Mieth Kriterien humaner Arbeitsverteilung und-beschaffung: Menschengerechte Arbeitsbedingungen müssen gewahrt werden (vgl. hierzu Kriterien humaner Arbeit bei Büssing/Aumann 1996, nach Rohmert, Hacker und Ulich, 136), Mitbestimmung muss gewährleistet sein, Arbeitsverteilung und -beschaffung muss umwelt- und friedensgerecht zugunsten betroffener Menschen vorgenommen werden, das Prinzip der Unteilbarkeit der Solidarität arbeitender Menschen mit benachteiligten Gruppen muss gewahrt bleiben.

Neben das Personalprinzip tritt daher das Solidaritätsprinzip. Mieth $(1999,89)$ umschreibt das Solidaritätsprinzip als "Option für beschädigtes Leben“. Sander (1996, 161ff.) verwendet seinen positiven Begriff der kreativen Widerständigkeit als Gütesiegel für gelingendes tätiges Leben. "Solidarität für die Güte des Lebens ist das entscheidende Merkmal des Schöpfungsgutes Arbeit.“ Ein entscheidendes Kriterium zur Umsetzung des Solidaritätsprinzipes stellt der gleichberechtigte Zugang zu Arbeit dar. Das von Mieth formulierte Recht auf Arbeit als moralisches Recht aus dem Grundrecht auf Selbstverwirklichung (Mieth 1999, 98) wird von Notz als das gerade für Frauen wohl am meisten in Frage gestellte Menschenrecht herausgestellt (Notz 1996, 100). Frauen sind von den "Schatten" der Arbeitsgesellschaft besonders betroffen. Der im Grundgesetz verankerte Gleichheitsgrundsatz hebt auch die Benachteiligung der Frauen im Erwerbsleben nicht auf. Sie werden nach den Anforderungen der aktuellen Arbeitsmarktlage ins Erwerbsleben gelockt, aus ihm vertrieben bzw. auf eine Biographie nach dem Dreiphasen-Schema festgelegt (Hengsbach 1990, 23). Sie werden - gerade nach dem Fall der Mauer - als eine der wesentlichen Ursachen für die Massenarbeitslosigkeit ausgemacht, sind tatsächlich allerdings qualitative Verliererinnen auf dem Arbeitsmarkt. Für sie ist weder der Grundsatz der Lohngleichheit noch der Grundsatz der eigenständigen Existenzsicherung umgesetzt (vgl. Notz 1996, 105ff.). Tätigkeiten, die als weiblich gelten oder traditionellerweise Frauen zugeschrieben werden, werden im Rahmen des fordisti- schen Produktions- und Wohlfahrtsmodells gering geschätzt (Senghaas-Knobloch 1999, 122).

Die konkrete Institutionalisierung zur Stützung einer gerechten Arbeitsteilung im Rahmen einer Debatte um einen neuen Gesellschaftsvertrag stellt sich als politische Notwendigkeit heraus. Wirtschaftliche Leistungen insgesamt sollen auf Männer und Frauen gerecht verteilt werden, Erwerbsarbeit sowie Nichterwerbsarbeit sollte von denen, die arbeiten können, gerecht und partnerschaftlich geleistet werden.

Bei Sander (1996, 161ff.) umfasst das Solidaritätsprinzip - als Solidarität für die Güte des Lebens - auch das Ökologieprinzip. Wir stellen das Ökologieprinzip mit Mieth, Müller, Senghaas-Knobloch, Ruh und Hengsbach u.a. als drittes politisch-ethisches Prinzip neben das Personal- und Solidaritätsprinzip.

Beim Umbau der Arbeitsgesellschaft wird die Beachtung des Ökologieprinzipes als sozialethisches Kriterium eine wesentliche Rolle spielen müssen. Die Natur wird im Rahmen der ökonomischen Rationalität als eigenständiger Produktionsfaktor weitgehend ausgeblendet. Der Raubbau an natürlichen Lebensgrundlagen zieht unüberschaubare ökologische Folgeschäden nach sich, die die Lebenschancen zukünftiger Generationen erheblich beeinträchtigt (vgl.Ökumenisch-sozialethischer Arbeitskreis NRW 2000, 12). Gerade die Nachhaltigkeit künftigen Wirtschaftens, d.h., die Schärfung des Bewusstseins für eine ökologisch verantwortliche Folgenabschätzung bei Problemlösungen, sollte in das Zentrum zukünftigen Wirtschaftens und Arbeitens gestellt werden. Das von Beherrschung und Unterwerfung der Natur geprägte Arbeitsverständnis ist von einem nachhaltigen, den Eigenwert der Natur beachtenden, Arbeitsverständnis abzulösen. Die Schaffung eines neuen Generationenvertrages vor dem Hintergrund der Mitgeschöpflichkeit des Menschen, seiner schöpfungsgemäßen Verantwortung für die Umwelt und für die nachfolgenden Generationen, sind in diesem Zusammenhang zentral (Mieth 1999, 96f.; Müller 1997, 82; Hengsbach 1999, 14; Ruh 1996, 209).

\subsection{Sozialethische Reflexion der Lösungsvorschläge}

Die in 2.1. entwickelten drei sozialethischen Prinzipien für den Umbau der Arbeitsgesellschaft sollen im Folgenden als Kriterien für die sozialethische Verträglichkeit der vorliegenden konkreten Handlungsansätze zum Wandel der Arbeitsgesellschaft angewandt werden. 
Gemäß dem oben entwickelten Personalprinzip soll der Mensch im Zentrum des Umbaus der Arbeitsgesellschaft stehen. Der Mensch besitzt Würde vor aller Arbeit und vor aller Tätigkeit. Mieth (1999, 100) schenkt dem Personalprinzip die notwendige Beachtung, indem er u.a. die eigene Selbstverwirklichung und die Vertiefung von Sinnerfahrungen als Hauptziele menschlicher Tätigkeit ausmacht. Die verbreitete Vorstellung der Entwicklung einer Zivilgesellschaft mit erweiterten Bürgerrechten für alle trägt dem Personalprinzip Rechnung. In der Zivilgesellschaft soll nicht primär der Markt, sondern die Menschen freigemacht werden (Ulrich 1997, 148). Auch Handlungsansätze, die eine Entkoppelung von Arbeit und Einkommen vorschlagen (Offe in Senghaas-Knobloch 1999, 129; Ulrich 1997, 149ff.), sei es durch die Einführung eines Grundeinkommens als Bürgergeld oder durch steuerliche Ausgleichszahlungen, verschaffen den Menschen die für die Entfaltung ihrer Persönlichkeit und Wahrung ihrer Menschenwürde angemessene Freiheit. Ansätze, die die Neuaufteilung der menschlichen Arbeitszeit befürworten und gleichzeitig den tätigen Menschen z.B. durch Fortbildung Weiterentwicklungen ermöglichen, fußen ebenfalls auf dem Personalprinzip (Mieth 1999, 104; Müller 1997, 83ff.; "New Work" in Senghaas-Knobloch 1999, 126).

Das Solidaritätsprinzip steht bei den Aufforderungen, vorhandene Arbeit und vorhandenes Einkommen zu teilen, im Mittelpunkt (Mieth 1999, 119; Müller 1997, 85; Möhring-Hesse 2000, 88). Dies gilt auch für Hengsbach $(1999,14)$, der gesellschaftlich nützliche Arbeit als Bemessungsgrundlage einer sozialen Sicherung sehen möchte. Eine die Aufteilung der menschlichen Arbeitszeit in Freizeit, Arbeitszeit und Sozialzeit begleitende Förderung der Sozialzeit (Ruh 1997, 4) unterstützt ebenfalls das Entstehen und Entfalten einer Solidargemeinschaft. Lösungsvorschläge, die auf die Entwicklung von Komplementärmärkten setzen (Ruh 1996, 207f.; Müller 1997, 84; Senghaas-Knobloch 1999, 125) oder der Ansatz von Giarini und Liedtke (vgl. Senghaas-Knobloch 1999, 128f.) können zur Entwicklung des Solidaritätsprinzipes beitragen, wenn gemeinnützige Kriterien wie Lebensdienlichkeit und Solidaritätsförderlichkeit formuliert und bei der Umsetzung der Ansätze beachtet werden.

Mieth $(1999,100)$ stellt das solidarische Moment direkt neben die personalen Elemente, wenn er als Hauptziele menschlicher Tätigkeit das Leben in gelingenden Beziehungen, sozialem Engagement und soziale Anerkennung formuliert. Alle Bemühungen, soziales Engagement aufzuwerten und Ge- meinwesenarbeit zu honorieren (Strasser 1999, 63), fußen auf dem Prinzip der Solidarität. Die Handlungsansätze, die um die Entkoppelung von Einkommen und Erwerbsarbeit kreisen, können die Entwicklung der Solidargemeinschaft fördern, indem sie zu einem Wertewandel weg von der Leistungsgerechtigkeit beitragen. Möhring-Hesse bringt die Wechselbeziehung zwischen Personal- und Solidaritätsprinzip auf den Punkt: "Die Koppelung von abhängiger Erwerbsarbeit und ,geregeltem Einkommen' kann genau in dem Maße aufgehoben werden, wie die Menschen, die nicht über Vermögen verfügen, anders als durch Erwerbsarbeit eigenständiges ,geregeltes Einkommen ' beziehen und vergleichbare Lebenslagen einnehmen sowie an der gesellschaftlich notwendigen Arbeit gleichberechtigt teilnehmen können." (Möhring-Hesse 2000, 105)

Folglich ist bei allen vorliegenden Handlungsansätzen zunächst die Wahrung des Personalprinzipes zu gewährleisten. Erst eine Solidargemeinschaft von freien Persönlichkeiten kann produktiv für die Gemeinschaft sein und so zurückwirken auf ihre Mitglieder. Insofern stehen die beiden ersten Prinzipien in einem wechselseitigen Abhängigkeitsverhältnis zueinander.

Das Ökologieprinzip steht im Zentrum derjeniger Ansätze, die um das Arbeiten in globaler und generationenübergreifender Verantwortung kreisen. Dazu gehören u.a. die Forderung von Mieth (1999, 102) nach einem übernationalen, die Umweltgerechtigkeit und Friedensgerechtigkeit ermöglichenden Weltarbeitskonsens oder Müllers (1997, 85) Forderung nach einem ökologischen Umbau durch die Förderung innovativer Umwelttechnologien. Notwendig für die Wahrung des Ökologieprinzipes ist, dass die sogenannten "global players" und die Wirtschaftsverbände ordnungspolitische Mitverantwortung für die Errichtung einer human-, sozialund umweltverträglichen Rahmenordnung des globalen Wettbewerbes im Rahmen einer Weltwirtschaftsordnung übernehmen (Ulrich 1997, 151). Der ökumenisch-sozialethische Arbeitskreis NRW konstatiert eine gefährliche ökonomische Naturvergessenheit, die noch heute übersieht, dass „WertSchöpfung" die Mitwirkung der Natur zur Voraussetzung hat. Umweltverträglichkeit sei notwendige Bedingung zukünftiger Gesellschaftsentwicklung. Es sei immer wieder die Frage zu stellen, inwieweit die Bedürfnisse der heutigen Generation befriedigt werden können, ohne die Lebensgrundlagen zukünftiger Generationen zu gefährden. (Ökumenischsozialethischer Arbeitskreis NRW 2000, 12). 


\section{Plädoyer für eine Einmischung der Sozialethik in den Umbau der Arbeitsgesellschaft}

Sicher war es kein Zufall, dass auf einer Tagung in der evangelischen Akademie Friedewald vom 15.17.03.2000 zum Thema "Logik der Ökonomie Krise der Arbeit" neben Friedhelm Hengsbach mit den Referenten Peter Ulrich und Hans Ruh zwei Schweizer Sozialethiker zum Thema sprachen. Womöglich erlebt man in der reformiert geprägten Schweiz die ethischen Dimensionen der Krise der Arbeitsgesellschaft schärfer als anderenorts und sucht deshalb nach Antworten. Auch kein Zufall dürfte die Quintessenz der Tagung, an denen GewerkschafterInnen und KirchenvertreterInnen teilnahmen, sein: Das Thema wächst von unten heran. Wenn dem so ist, dass die Thematik von Beschäftigten, Initiativen und politischen Bewegungen auf die Tagesordnung gesetzt wird - ist es an der Zeit, dass die Sozialethik sich bewegt und Beiträge formuliert für die Mitarbeit an einer neuen Gesellschaft, die aus den Trümmern der Arbeitsgesellschaft (Gorz) entstehen soll.

Als Beispiel für ein sozialethisches Erarbeiten des Themenkomplexes, ,von unten“ "können die theologisch-ethischen Impulse für eine menschengerechte Gesellschaftsentwicklung des ökumenischsozialethischen Arbeitskreises NRW gelten (Ökumenisch-sozialethischer Arbeitskreis NRW 2000, 10-20). Der Arbeitskreis sieht sich mit seinen Thesen in der Tradition des Sozialwortes der beiden Kirchen, wenn er ausgehend von der Beteiligungsprämisse die Grenzen des traditionellen Verständnisses von Erwerbsarbeit analysiert und den Versuch einer Neubestimmung des Verhältnisses von Arbeit, Einkommen und Leben vornimmt. Dabei wird eine sozialethische Profilierung des Gerechtigkeitsbegriffes vorgelegt, der die Wahrung der Teilhabe-, Leistungs- und Verfahrensgerechtigkeit in dieser hierarchischen Reihenfolge vorschlägt. Darüber hinaus sind alle Arbeitsformen und Tätigkeitsbereiche vor dem Leitbild der ,Nachhaltigkeit“ zu messen. (Ökumenisch-sozialethischer Arbeitskreis NRW 2000, 13-17)

Die Frage, warum sich aber weiterhin das Gros der sozialethischen DenkerInnenschaft aus dieser gesellschaftlich relevanten Debatte heraushält, gründet in der Frage nach der heutigen Funktion von Sozialethik. Was will sie letztlich bewirken? Liegt ihre Aufgabe in der internen Debatte auf metaethischer Ebene oder darf und muss sie sich sogar in eine laufende politische Debatte einmischen? Kissling weist der Sozialethik eine dreigeteilte künftige Rol- le zu: Sie soll advokatorisch die Ansprüche der praktischen Vernunft gegenüber der Mittel/ZweckRationalität und die Ansprüche des Gemeinwohls gegenüber den Partikularinteressen vertreten. Sie soll katalysatorisch die Ansprüche des menschenund umweltgerechten Handelns in den Vordergrund rücken. Und sie soll letztlich kooperativ mit all denen arbeiten, die den Sinn für die Unterscheidung zwischen legitimen und illegitimen Ansprüchen behalten haben (Kissling 1994, 214). Dann aber muss sie über die Postulierung von Maxima hinaus an der politischen Umsetzung Status quo bezogener Minima mitwirken. Dabei können die Überlegungen von Möhring-Hesse (2000, 93ff.) zu einer Gesellschaftsethik allgemeiner Beteiligung weiterführen. Da nicht unmittelbar die Erwerbsarbeit sondern Beteiligung die normative Prämisse einer solchen Ethik ist, braucht sie nicht bei der Forderung nach einer Verallgemeinerung der Erwerbsarbeit stehen zu bleiben sondern kann in Antwort auf die Krise der Arbeitsgesellschaft über diese hinausdenken (2000, 105). Sie kann Relativierungen und Korrekturen arbeitsgesellschaftlicher Strukturen kenntlich machen - auch wenn noch kein überzeugendes Konzept des Ausstiegs aus der Arbeitsgesellschaft, das vergleichbare Lebens- und Beteiligungsmöglichkeiten für alle sichern hilft, statt die soziale Spaltung zu verschärfen, erkennbar ist $(2000,110)$. Der Einsatz auf programmatischer Ebene und vor Ort, z.B. in den regionalen Bündnissen für Arbeit, ist eine elementare Aufgabe einer handlungsorientierten Sozialethik.

\section{Literatur}

Büssing, André; Sandra Aumann (1996): Telearbeit aus arbeitspsychologischer Perspektive; in: ARBEIT 2, 133-153

Einheitsübersetzung (1980). Freiburg, Basel, Wien

Evangelische Kirche in Deutschland (1991): Evangelisches Bildungsverständnis in einer sich wandelnden Arbeitsgesellschaft; in: Kirchenamt der EKD (Hg.): EKD-Texte 37

Evangelische Kirche in Deutschland/Sekretariat der Deutschen Bischofskonferenz (Hg.) (1991): Für eine Zukunft in Solidarität und Gerechtigkeit. Wort der Evangelischen Kirche in Deutschland und der Deutschen Bischofskonferenz zur wirtshaftlichen und sozialen Lage in Deutschland (Gemeinsame Texte 9), Bonn

Foitzik, Alexander (1996): Was ist Arbeit wert?; in: HK $2,55-57$

Gorz, André (2000): Arbeit zwischen Misere und Utopie, Frankfurt 
Guggenberg, Bernd (1999): Arbeit und Lebenssinn; in: Gerd Haeffner u.a.: Arbeit im Umbruch. Sozialethische Maßstäbe für die Arbeitswelt von morgen. Stuttgart, 123-144

Hank, Rainer (1995): Arbeit - Die Religion des 20. Jahrhunderts. Auf dem Weg in die Gesellschaft der Selbständigen. Frankfurt (M.)

Hengsbach, Friedhelm (1990): Strukturentgiftung: Kirchliche Soziallehre im Kontext von Arbeit, Umwelt, Weltwirtschaft. Düsseldorf

Hengsbach, Friedhelm (1999): Die Zukunft der Arbeit ist nicht ihr Ende; in: Orientierung 2, 13-15

Honecker, Martin (1995): Grundriss der Sozialethik. Berlin/New York

Kissling, Christian (1994): Die Funktion der Sozialethik; in: Orientierung 19, 210-214

Mieth, Dietmar (1999): Sozialethische Reflexionen zur Abeit im Umbruch; in: Gerd Haeffner u.a.: Arbeit im Umbruch. Sozialethische Maßstäbe für die Arbeitswelt von morgen. Stuttgart, 85-106

Möhring-Hesse, Matthias (2000): Arbeit integriert Arbeit spaltet. Sozialkatholische Reflexionen über gesellschaftliche Integration und Erwerbsarbeit; in: Heiner Ludwig, Karl Gabriel (Hg.): Gesellschaftliche Integration durch Arbeit. Über die Zukunftsfähigkeit sozialkatholischer Traditionen von Arbeit und Demokratie am Ende der Industriegesellschaft. Münster, 81-112

Müller, Johannes (1997): Arbeitslosigkeit als globale Herausforderung. Sozialethische Maßstäbe für die Arbeitswelt von heute und morgen; in: Stimmen der Zeit 2, 75-86

Notz, Gisela (1996): Die Auseinandersetzung dauert an - Frauen im Kampf um Arbeit und Menschenwürde; in: Oswald-von-Nell-Breuning-Haus (Hg.): Arbeit und Menschenwürde. Standpunkte, Kontexte, Perspektiven. Bornheim, 99-128

Ökumenisch-sozialethischer Arbeitskreis NRW (2000): Auf dem Weg zu einer Neubestimmung von Arbeit, Einkommen und Leben - Thesen zur Arbeit der Zukunft, in: Sozial Katholischer Informationsdienst 3/2000, 10-20

Rich, Arthur (1990): Wirtschaftsethik Bd. 2: Marktwirtschaft, Planwirtschaft, Weltwirtschaft aus sozialethischer Sicht. Gütersloh

Ruh, Hans (1996): Arbeitsmarkt und Sozialstat - Eine Thesenreihe; in: Zeitschrift für evangelische Ethik 40, 205-210

Sander, Hans-Joachim (1996): Arbeit - die Humanisierungstechnik der Schöpfung. Die religiöse Widerständigkeit eines Produktionsfaktors; in: Oswaldvon-Nell-Breuning-Haus (Hg.): Arbeit und Menschenwürde. Standpunkte, Kontexte, Perspektiven. Bornheim, 153-175

Senghaas-Knobloch, Eva (1999): Von der Arbeits- zur Tätigkeitsgesellschaft? Zu einer aktuellen Debatte; in: ARBEIT 2, 117-136
Strasser, Johano (1999): Wenn der Arbeitsgesellschaft die Arbeit ausgeht. Zürich

Ulrich, Peter (1997): Arbeitspolitik jenseits des neoliberalen Ökonomismus - das Kernstück einer lebensdienlichen Sozialpolitik; in: Franz Furger (Hg.): Jahrbuch für christliche Sozialwissenschaften 38. Münster, 136-152

Zimmerli, Walther Ch. (1997): Vergangenheit und Zukunft der Arbeit. Ethische Perspektiven, in: Hauptverband der gewerblichen Berufsgenossenschaften (HVBG) (Hg.): Dokumentation Symposium "Zukunft der Arbeit" 1996, St. Augustin, 143-164

Dr. Manfred Körber

Bischöfliches Generalvikariat des Bistums Aachen Klosterplatz 7

D-52062 Aachen

Dipl. Theol. Jolanda Staman

Forum der Arbeit

Wiesenstr. 17

D-52134 Herzogenrath 